\title{
Photocount statistics of the Josephson parametric amplifier
}

\author{
Jean Olivier Simoneau, ${ }^{1,{ }^{*}}$ Stéphane Virally $\odot,{ }^{1,2}$ Christian Lupien, ${ }^{1}$ and Bertrand Reulet ${ }^{1}$ \\ ${ }^{1}$ Institut Quantique, Département de Physique, Université de Sherbrooke, Sherbrooke, Québec, Canada J1K $2 R 1$ \\ ${ }^{2}$ Départment de Génie Physique, Polytechnique Montréal, Montréal, Québec, Canada H3T 1J4
}

(Received 28 October 2020; revised 23 September 2021; accepted 31 January 2022; published 4 March 2022)

\begin{abstract}
Parametric amplifiers are known to squeeze the vacuum state of the electromagnetic field, thus the statistics of the photocounts at their output should be that of squeezed vacuum. However, several theoretical works predict a very different statistical distribution. We have measured the photocount statistics of a Josephson parametric amplifier and recover the expected squeezed vacuum statistics. We explain this discrepancy by showing theoretically how the photocount statistics is dictated by the detection process. Namely, most experiments will naturally measure a single mode of the electromagnetic field, while the aforementioned theoretical works implicitly describe massively multimode detection.
\end{abstract}

DOI: 10.1103/PhysRevResearch.4.013176

\section{INTRODUCTION}

From an experimental perspective, a photon can be described as two causally linked events, a creation and an annihilation. The statistics of photocounts must then depend both on the emission and the detection modes, and predictions about statistics of photons emitted by any system should always specify the detection setup.

This fact becomes an important factor in some experiments. Consider, for instance, the output of a Josephson parametric amplifier (JPA) [1-5]. This type of device is very much at the forefront of quantum optics in microwaves, as it constitutes a quantum-limited amplifier in this band and as such is likely to be used in all quantum computing and measurement schemes. Understanding the noise characteristics of those devices is critical for these typically small-signal applications. The JPA is fundamentally the equivalent in the microwave domain of the optical parametric oscillator (OPO), for which the statistics of photocounts was computed as early as 1989 [6], although the variance was not explicitly computed at the time. A more recent paper [7] predicts the full counting statistics of photocounts emitted by a JPA with an electromagnetic vacuum input. The result is the same as that of the previous work [6], and the variance is explicitly given as $\left\langle\delta n^{2}\right\rangle=2\langle n\rangle\left[\left(8 / \gamma^{2} \tau^{2}\right)\langle n\rangle^{2}+(5 / \gamma \tau)\langle n\rangle+1\right]$, where $\gamma$ is the width of the cavity and $\tau$ the integration time for each count. This result appear to contradict the model of parametric amplifiers as "vacuum squeezers," a well-studied quantum optics fact. The squeezing operator generates pairs of photons,

\footnotetext{
"Present address: Nord Quantique, Sherbrooke, Québec, Canada J1K 0A5; Jean.Olivier.Simoneau@USherbrooke.ca

Published by the American Physical Society under the terms of the Creative Commons Attribution 4.0 International license. Further distribution of this work must maintain attribution to the author(s) and the published article's title, journal citation, and DOI.
}

and this is reflected in the photocount variance, which reads $\left\langle\delta n^{2}\right\rangle=2\langle n\rangle(\langle n\rangle+1)$ [8]. Both formulas agree at very small signal, yielding $\left\langle\delta n^{2}\right\rangle \simeq 2\langle n\rangle$, twice the classical value. This reflects the emission of pairs of photons in the squeezing process. However, the variance of Ref. [7] dramatically increases as $\langle n\rangle^{3}$ for higher signals. This is a strong departure form the expected behavior of a squeezed vacuum.

In this paper, we show that the apparent discrepancy is actually due to the choice of detection scheme in the theoretical works. Namely, the detector is assumed to have infinite frequency resolution. One consequence is that a very large (theoretically infinite) number of distinct modes of the electromagnetic field are measured: The detection is massively multimode, which impacts the statistics of photocounts, a result already known in quantum optics [9-11]. Of course, real measurements are limited both in time and bandwidth. They inherently possess finite frequency resolution. More importantly, detection will usually be performed in a relatively narrow band around the cavity of the parametric amplifier, to remove most of the noise. Such a detection scheme will naturally measure a single mode of the electromagnetic field. Here, we show both theoretically and experimentally that the expected squeezed vacuum statistics is recovered in the latter case.

The paper is organized as follows. We present an experiment with limited frequency resolution at the output of a JPA with vacuum input. In contrast with quantum optics, the discrete photocount statistics is recovered from continuous voltage measurements [12]. We show that after careful calibration, we recover a variance and third-order photocount moment equal to those predicted for a squeezed vacuum, and not those predicted by Refs. [6,7]. These measurements are well captured by a simple input-output [13-15] model of the JPA, followed by a single-mode (non-frequency-resolved) detector. In contrast, a variant of the model, using the same input-output relations for the JPA but a multimode (frequencyresolved) detector, leads to the statistics predicted by Ref. [7]. The distinction is lost in narrow-band experiments, but we 


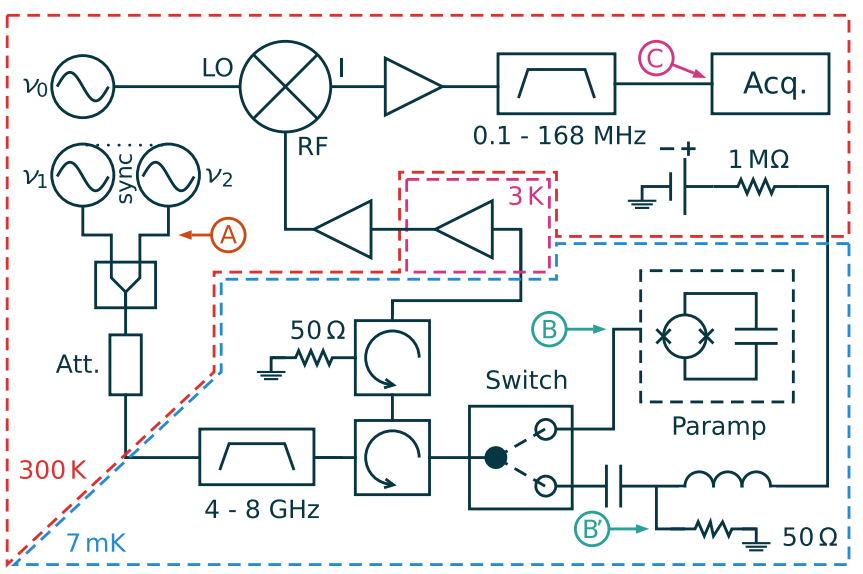

FIG. 1. Experimental setup used for detection. The flux bias coil of the paramp is omitted for clarity. Circled letters are calibration reference points. See text for details.

anticipate that it will play a crucial role in the results of future experiments using the new generation of wide bandwidth JPAs $[16,17]$.

\section{EXPERIMENTAL SETUP}

The experimental setup is presented in Fig. 1. We study the signal emitted by a commercial Josephson parametric amplifier (paramp), similar to that of Ref. [18], placed in a dilution refrigerator at $\sim 7 \mathrm{mK}$ and driven by two sinusoidal pumps of frequencies $v_{1}=4.5 \mathrm{GHz}$ and $v_{2}=7.5 \mathrm{GHz}$. The output signal is measured in a small frequency band centered around $\left(v_{1}+v_{2}\right) / 2=6.0 \mathrm{GHz}$. The dual-pump operation mode [19] is selected to avoid residual pump signal in the measurement band, so that the input of the paramp in the measured bandwidth can be considered as the vacuum.

The paramp resonance frequency can be tuned by a current bias through a superconducting flux coil in the vicinity of the paramp superconducting quantum interference device (SQUID) loop (omitted on the schematic for clarity). A 4$8 \mathrm{GHz}$ bandpass filter protects the paramp from radiation outside of its operation range. Circulators are used to separate the input and output fields of the paramp and to isolate it from the noise of the 3 - and $300-\mathrm{K}$ stages. A microwave switch is used to swap a $50-\Omega$ resistor in place of the paramp for calibration purposes.

The paramp output signal is amplified and conveyed to $300 \mathrm{~K}$, where it is downconverted by an in-phase/quadrature (IQ) mixer with a local oscillator $(\mathrm{LO})$ at frequency $v_{0} \approx$ 6.0 GHz. The LO is not phase locked with the pumps. The downconverted signal is then filtered by a $0.1-168 \mathrm{MHz}$ bandpass filter and sampled by a fast acquisition card with 14-bit resolution and $400 \mathrm{MSa} / \mathrm{s}$ rate. Histograms of the measured signal are accumulated and their first six cumulants are computed on the fly during the data acquisition. Each data point corresponds to $11.5 \times 10^{9}$ voltage measurements.

\section{CALIBRATION}

Proper calibration is essential to compare experimental results with theory. Three calibrations are required: that of the ac power at the sample level, that of the absolute photon numbers that are detected, calculated for the measured voltage cumulants $C_{j}$, and that of the paramp resonance frequency versus current in the flux bias coil.

To calibrate the attenuation between the excitation at room temperature (circle reference $A$ in Fig. 1) and the input port of the paramp $\left(\mathrm{B} \approx \mathrm{B}^{\prime}\right.$ ), we use a macroscopic $R=50 \Omega$ resistor in place of the paramp (using the cryogenic switch). We can heat that resistor using either a known dc current or an ac bias and observe the temperature increase by the increased noise it emits. Thus we can map which dc current is needed to heat the resistor as much as a given ac voltage, as in Ref. [20]. The linear relation we observe between them provides us with the A-B attenuation, $62.7 \mathrm{~dB}$.

To calibrate the effective gain between the output of the paramp (B) and the data acquisition $(C)$, we measure the $A-C$ gain by adjusting the paramp dc flux line to put it out of resonance such that it totally reflects an incoming test tone signal of known amplitude, and subtract the previously obtained A-B attenuation. We find the $\mathrm{B}-\mathrm{C}$ gain to be $87.7 \mathrm{~dB}$.

The paramp resonance frequency, which is controlled by the current applied to the flux bias coil, is calibrated by measuring the reflected phase on the paramp using a vector network analyzer in the absence of a pump signal and for very weak excitation [18,21]. The same measurement also provides the resonance width $\gamma \sim 135 \mathrm{MHz}$.

\section{MEASUREMENTS}

In order to probe the photon statistics of the paramp for different regimes of operations, we explore its parameter space, flux bias, and pump power, for a fixed measurement frequency $v_{0}=\left(v_{1}+v_{2}\right) / 2=6.0 \mathrm{GHz}$. Experimentally, we first select a pump power yielding a maximum gain of approximately $10 \mathrm{~dB}$ and adjust the paramp at this operation point. Then, we sweep the flux bias current and the pump power around the initial values while measuring the even cumulants $C_{2 k}=\left\langle\left\langle V^{2 k}\right\rangle\right\rangle$ of the voltage fluctuations generated by the paramp, shown in Fig. 2 for $k=1,2$, and 5 for $k=3$. From these we compute the unitless cumulants $C_{2 k} /\left(Z B h v_{0}\right)^{k}$, with $Z=50 \Omega$, from which we deduce the moments $\left\langle\delta n^{k}\right\rangle$ of the photocount distribution, using the procedure developed in some of our previous works [12,22]. We show in Fig. 3 the variance $(k=2)$ of the photocount distribution as a function of the average photon number. The third-order moment is treated in Appendix C (see Figs. 5 and 6). There are actually many combinations of flux bias and pump power that give the same average photon number $\langle n\rangle$ but different values of $\left\langle\delta n^{2}\right\rangle$. As a consequence, Fig. 3 exhibits clouds of experimental points and not just a single curve. A particular subset of points corresponds to the maximum gain of the paramp, i.e., the largest value of $\langle n\rangle$ for each pump power. Those are the best operating points for the paramp used as an amplifier; they are represented as blue solid points in Fig. 2(a). Reporting these points in Fig. 2(b), we observe that they are close to the maximum of the fourth cumulant $C_{4}$. In Appendix $C$, we also show that they also correspond to a vanishing $C_{6}$. The same points are highlighted in Fig. 3 (open circles). We find that these specific points closely follow the expected relations for a squeezed vacuum, represented by dashed lines in Figs. 3 

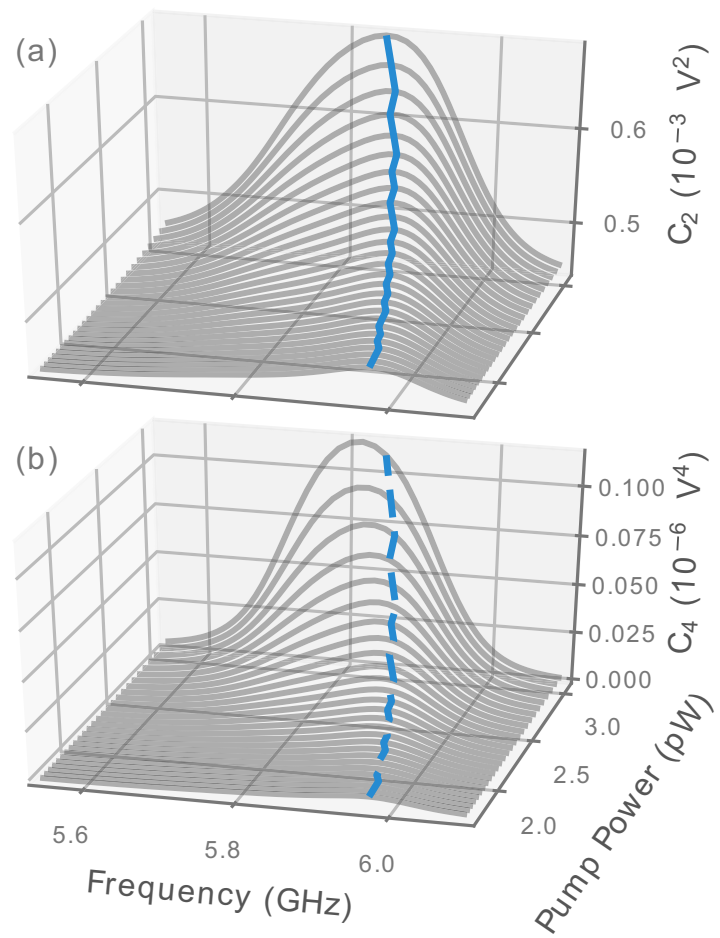

FIG. 2. Measured cumulants of voltage fluctuations generated by the paramp as a function of its resonance frequency and pump power. The Frequency axis is controlled by the flux bias. The thick blue solid line in (a) corresponds to the ridge of $C_{2}$. The dashed blue line in (b) corresponds to frequency and pump power equivalent to the blue line of (a).

and 6. We discuss why theory is best obeyed for these points in Appendix C.

\section{THEORY}

To model the JPA, we apply the input-output formalism [13-15] detailed in Appendix A to a single-ended,

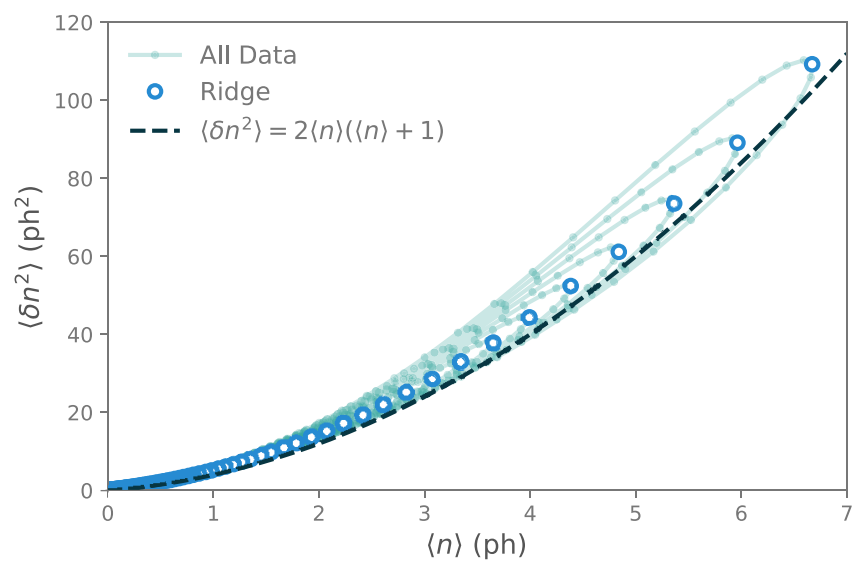

FIG. 3. Variance of the photocounts $\left\langle\delta n^{2}\right\rangle$ as a function of the average photon number $\langle n\rangle$. Dots are experimental data and each line represents a given pump power. Open circles correspond to the maximum of the paramp gain, i.e., the blue line in Fig. 2. The dashed line corresponds to the theoretical prediction for squeezed vacuum. frequency-symmetric single-mode cavity. The intracavity Hamiltonian is assumed to be the squeezing Hamiltonian that is characteristic of parametric amplifiers [23-26]. The output electromagnetic modes can be written as a function of the free input modes $\boldsymbol{b}_{v}$, in the frame rotating at $v_{0}$ and up to a constant phase, as

$$
\boldsymbol{B}_{\text {out }}(v)=\cosh [\eta(v)] \boldsymbol{b}_{v}+e^{i \theta} \sinh [\eta(v)] \boldsymbol{b}_{-v}^{\dagger},
$$

with $\theta$ defining a squeezing direction, and $\eta(v)$ a squeezing strength defined in Eq. (A26).

The photon-photon interaction Hamiltonian also shifts the position of the center peak of the cavity mode by $|\xi|\left(P_{1}+\right.$ $\left.P_{2}\right) / \sqrt{P_{1} P_{2}}$, where $\xi$ is the nonlinear coupling inside the cavity and $P_{1}$ and $P_{2}$ are the respective pump powers. This effect is clearly observed in Fig. 2. The peak can be brought back to the center of the measurement window by adjusting a magnetic flux $\phi$. The "ridge" (maximum) of $C_{2}$ observed in Fig. 2 corresponds to the condition where $\phi$ exactly cancels the frequency shift.

Using this model, we calculate the moments of the statistics of the photon flux per unit of frequency and time,

$$
\begin{aligned}
\left\langle n^{k}\right\rangle= & \int d v_{1} \cdots d v_{2 k} h^{*}\left(v_{1}\right) h\left(v_{2}\right) \cdots h^{*}\left(v_{2 k-1}\right) h\left(v_{2 k}\right) \\
& \times\left\langle\phi_{\mathrm{i}}\left|\boldsymbol{B}_{\text {out }}^{\dagger}\left(v_{1}\right) \boldsymbol{B}_{\text {out }}\left(v_{2}\right) \cdots \boldsymbol{B}_{\text {out }}^{\dagger}\left(v_{2 k-1}\right) \boldsymbol{B}_{\text {out }}\left(v_{2 k}\right)\right| \phi_{\mathrm{i}}\right\rangle,
\end{aligned}
$$

for a detector with normalized response function $h(v)$, with $\left|\phi_{\mathrm{i}}\right\rangle$ the input state of the JPA. The response function $h(v)$ characterizes the single mode of the electromagnetic field being detected. In our case, it is well approximated by a square window of width $B=2 \times 168 \mathrm{MHz}$, corresponding to the response of the $0.1-168 \mathrm{MHz}$ bandpass filter applied to the downconverted signal (see the experimental setup section above). The effective photocount integration time is thus $\tau=1 / B$. In the case of an electromagnetic vacuum input, $\left|\phi_{\mathrm{i}}\right\rangle=|\mathrm{vac}\rangle$, we find the expected statistics of a squeezed vacuum on the "ridge" of $C_{2}$. In particular, for $\eta(v)$ in the unit range or above, as detailed in Appendix B,

$$
\langle n\rangle=\int d v|h(v)|^{2} n(v),
$$

with $n(v)=\sinh ^{2}[\eta(v)]$, and

$$
\left\langle\delta n^{2}\right\rangle=2\langle n\rangle(\langle n\rangle+1) .
$$

The Fano factor $\mathcal{F}=\left\langle\delta n^{2}\right\rangle /\langle n\rangle$ is thus simply

$$
\mathcal{F}=2(\langle n\rangle+1) \text {. }
$$

This result is at odds with the predictions of Ref. [7]. The reason is that the theoretical framework of the reference uses a different detection scheme, where the signal is resolved in frequency. For a frequency resolution $\Delta$, the measured moments per unit time are

$$
\begin{aligned}
\left\langle n^{k}\right\rangle_{\Delta}= & \int d v_{1} \cdots d v_{2 k} \delta_{\Delta}\left(v_{1}-v_{2}\right) \cdots \delta_{\Delta}\left(v_{2 k-1}-v_{2 k}\right) \\
& \times\left\langle\phi_{\mathrm{i}}\left|\boldsymbol{b}_{\mathrm{o}}^{\dagger}\left(v_{1}\right) \boldsymbol{b}_{\mathrm{o}}\left(v_{2}\right) \cdots \boldsymbol{b}_{\mathrm{o}}^{\dagger}\left(v_{2 k-1}\right) \boldsymbol{b}_{\mathrm{o}}\left(v_{2 k}\right)\right| \phi_{\mathrm{i}}\right\rangle
\end{aligned}
$$

where $\delta_{\Delta}$ are peaked functions of width $\Delta$ and unit integrated value. They tend to the true Dirac delta distribution as $\Delta \rightarrow 0$ 
(see Appendix B). In the same limit, the Fano factor $\mathcal{F}_{\Delta} \equiv$ $\left\langle\delta n^{2}\right\rangle_{\Delta} /\langle n\rangle_{\Delta}$ behaves as

$$
\lim _{\Delta \rightarrow 0} \mathcal{F}_{\Delta}=\frac{\int d \nu 2 n(\nu)[n(\nu)+1]}{\int d \nu n(v)} .
$$

This result is very different from that of Eq. (4). It corresponds to summing many independent modes, resolved in frequency. Each mode is a squeezed vacuum with a Fano factor of the form of Eq. (5). As a consequence, the behavior of the overall Fano factor is dependent on the detector bandwidth.

\section{CONCLUSION}

We have performed an experimental and theoretical investigation of the photon statistics of the microwave radiation generated by a Josephson parametric amplifier. We have observed that with a wideband, single-mode detection scheme, the statistics is that of squeezed vacuum when the pump of the paramp is kept to its lowest value for a given average photon number. Our theoretical analysis shows how the photocount statistics crucially depends on the detection bandwidth, from a time-resolved, wideband amplifier (our setup) to that of frequency-resolved photodetection [6,7]. Our results, which are valid for any kind of paramp, are of great interest both to the development of quantum-limited amplifiers with optimal photon statistics as well as for the development of sources of radiation with nonclassical statistics. As a matter of fact, instead of playing with the source, we show that one can play with the detector, in a similar way as quantum computation may require non-Gaussian states of light if measurements are performed with linear detectors whereas Gaussian states are enough if one uses single-photon detectors [27]. More theoretical and experimental works are needed to explore the path we have paved, in particular to understand how high-order terms in the Hamiltonian affect the photocount distribution for an arbitrary detection bandwidth.

\section{ACKNOWLEDGMENTS}

We thank G. Laliberté for technical help and S. Boutin for fruitful discussions. This work was supported by the Canada Excellence Research Chair program, the NSERC, the Canada First Research Excellence Fund, the MDEIE, the FRQNT, the INTRIQ, the Université de Sherbrooke, and the Canada Foundation for Innovation.

\section{APPENDIX A: INPUT-OUTPUT MODEL}

We consider a single-sided, narrow-band cavity mode $\boldsymbol{A}(t)$ coupled on one side to a continuum of modes $\boldsymbol{B}_{v}(t)$. The coupling coefficient $\gamma$ is assumed to be constant over the narrow range of frequencies under consideration. The resonance of the cavity can be modified via a magnetic flux $\phi$. The cavity is pumped by two narrow-band signals with power $P_{1}$ and $P_{2}$ and respective frequencies $v_{1}$ and $\nu_{2}$. We detect the output signal in a narrow band around $v_{0}=\left(v_{1}+v_{2}\right) / 2$.

We use the input-output formalism [13-15] to model the interaction between the cavity mode and the output modes [26].
Evolution of the external operators. The Hamiltonian for the external modes is

$$
\boldsymbol{H}_{b}=\int_{-\infty}^{+\infty} d \nu 2 \pi \hbar v \boldsymbol{B}_{v}^{\dagger} \boldsymbol{B}_{v},
$$

while the interaction Hamiltonian is

$$
\boldsymbol{H}_{i}=i \hbar \sqrt{\gamma} \int_{-\infty}^{+\infty} d \nu\left[\boldsymbol{B}_{v}^{\dagger} \boldsymbol{A}-\boldsymbol{B}_{v} \boldsymbol{A}^{\dagger}\right] .
$$

Strictly speaking, $v$ only goes from 0 to $+\infty$. However, as usual in quantum optics [13] in the narrow-band regime, i.e., when the modes are centered around a frequency $v_{0}$ and in a bandwidth $B \ll v_{0}$, we extend the limits of all integrations in $v$ from $-\infty$ to $+\infty$.

The $\boldsymbol{B}_{v}$ operators evolve as

$$
\frac{d \boldsymbol{B}_{v}}{d t}=\frac{i}{\hbar}\left[\boldsymbol{H}_{b}+\boldsymbol{H}_{i}, \boldsymbol{B}_{v}\right]
$$

and we find

$$
\begin{aligned}
\boldsymbol{B}_{v}(t) & =\boldsymbol{B}_{v}\left(t_{\text {in }}\right) e^{-i 2 \pi v\left(t-t^{\text {in }}\right)}+\sqrt{\gamma} \int_{t^{\text {in }}}^{t} d \tau \boldsymbol{A}(\tau) e^{-i 2 \pi v(t-\tau)} \\
& =\boldsymbol{B}_{v}\left(t_{\text {out }}\right) e^{-i 2 \pi v\left(t-t^{\text {out }}\right)}-\sqrt{\gamma} \int_{t}^{t^{\text {out }}} d \tau \boldsymbol{A}(\tau) e^{-i 2 \pi v(t-\tau)},
\end{aligned}
$$

where $t_{\text {in }}$ and $t_{\text {out }}$ are times before and after interaction of the external modes with the cavity.

We define the integrated external modes

$$
\begin{aligned}
\boldsymbol{B}(t) & =\int_{-\infty}^{+\infty} d v \boldsymbol{B}_{v}(t), \\
\boldsymbol{B}_{\text {in }}(t) & \equiv-\int_{-\infty}^{+\infty} d v \boldsymbol{B}_{v}\left(t_{\text {in }}\right) e^{-i 2 \pi v\left(t-t_{\text {in }}\right)} \\
& \equiv \int_{-\infty}^{+\infty} d v \boldsymbol{B}_{\text {in }}(v) e^{-i 2 \pi v t}, \\
\boldsymbol{B}_{\text {out }}(t) & \equiv+\int_{-\infty}^{+\infty} d v \boldsymbol{B}_{v}\left(t_{\text {out }}\right) e^{-i 2 \pi v\left(t-t_{\text {out }}\right)} \\
& \equiv \int_{-\infty}^{+\infty} d v \boldsymbol{B}_{\text {out }}(\nu) e^{-i 2 \pi v t},
\end{aligned}
$$

where the \pm signs are only a convention. This leads to the input-output relations between the external modes before and after interaction,

$$
\boldsymbol{B}(t)=-\boldsymbol{B}_{\text {in }}(t)+\frac{\sqrt{\gamma}}{2} \boldsymbol{A}(t)=\boldsymbol{B}_{\text {out }}(t)-\frac{\sqrt{\gamma}}{2} \boldsymbol{A}(t) .
$$

We assume that the input modes have not interacted with anything. That is, we identify

$$
\boldsymbol{B}_{\text {in }}(v)=\boldsymbol{b}_{v},
$$

where the $\boldsymbol{b}_{v}$ are the free modes of the electromagnetic (EM) field.

Evolution of the cavity operator. The Hamiltonian for the internal mode is $\boldsymbol{H}_{a}$. It evolves as

$$
\frac{d \boldsymbol{A}}{d t}=\frac{i}{\hbar}\left[\boldsymbol{H}_{a}+\boldsymbol{H}_{i}, \boldsymbol{A}\right] .
$$


For a bare, narrow-band cavity

$$
\boldsymbol{H}_{a}=2 \pi \hbar v_{0} \boldsymbol{A}^{\dagger} \boldsymbol{A},
$$

leading to

$$
\begin{aligned}
\frac{d \boldsymbol{A}}{d t} & =\left(-i 2 \pi v_{0}-\gamma / 2\right) \boldsymbol{A}+\sqrt{\gamma} \boldsymbol{B}_{\text {in }} \\
& =\left(-i 2 \pi v_{0}+\gamma / 2\right) \boldsymbol{A}-\sqrt{\gamma} \boldsymbol{B}_{\text {out }} .
\end{aligned}
$$

We first eliminate $v_{0}$ by placing ourselves in the rotating frame. In addition, we define the Fourier transform of $\boldsymbol{A}(t)$,

$$
\boldsymbol{A}(v)=\int_{-\infty}^{+\infty} d t \boldsymbol{A}(t) e^{i 2 \pi v t}
$$

and obtain

$$
\begin{gathered}
(-i 2 \pi v+\gamma / 2) \boldsymbol{A}(v)=\sqrt{\gamma} \boldsymbol{b}_{v}, \\
(i 2 \pi v+\gamma / 2) \boldsymbol{A}(v)=\sqrt{\gamma} \boldsymbol{B}_{\text {out }}(v) .
\end{gathered}
$$

Note that we have replaced $\boldsymbol{B}_{\text {in }}(v)$ by $\boldsymbol{b}_{v}$ in the first equality.

For a cavity with an infinite $Q$ factor, we would expect

$$
\boldsymbol{A}(v)=e^{i \phi(v)} \sqrt{\delta(v)} \boldsymbol{b}_{v} .
$$

Indeed, we get

$$
\boldsymbol{A}(v)=\frac{2 \sqrt{\gamma}}{\gamma / 2-i 2 \pi v} \boldsymbol{b}_{v}
$$

so that for $\gamma \rightarrow \infty$, the expression indeed tends towards the square root of the delta distribution.

We then find

$$
\boldsymbol{B}_{\text {out }}(\nu)=e^{-i 2 \arctan (4 \pi v / \gamma)} \boldsymbol{b}_{v},
$$

which shows that the output modes remain the free modes of the EM field, with a frequency-dependent phase, as expected. The input-output relation is unitary.

For a narrow-band parametric amplifier, we have

$$
\boldsymbol{H}_{a}=2 \pi \hbar\left(v_{0}+\delta\right) \boldsymbol{A}^{\dagger} \boldsymbol{A}+i 2 \pi \hbar\left(\xi \boldsymbol{A}^{\dagger} \boldsymbol{A}^{\dagger}-\xi^{*} \boldsymbol{A} \boldsymbol{A}\right),
$$

where $\xi$ is the nonlinear coefficient of the amplification process and $\delta=\phi+|\xi|\left(P_{1}+P_{2}\right) / \sqrt{P_{1} P_{2}}(\phi$ is the frequency displacement provided by the magnetic flux).

We eliminate $v_{0}$ by placing ourselves in the rotating frame, and get

$$
\begin{aligned}
& \frac{i}{\hbar}\left[\boldsymbol{H}_{a}, \boldsymbol{A}(t)\right]=-i 2 \pi \hbar \delta \boldsymbol{A}(t)+2 \pi \xi \boldsymbol{A}^{\dagger}(t), \text { or } \\
& \frac{i}{\hbar}\left[\boldsymbol{H}_{a}, \boldsymbol{A}(v)\right]=-i 2 \pi \delta \boldsymbol{A}(v)+2 \pi \xi \boldsymbol{A}^{\dagger}(-v),
\end{aligned}
$$

as

$$
\begin{aligned}
\boldsymbol{A}^{\dagger}(t) & =\int_{-\infty}^{+\infty} d v \boldsymbol{A}^{\dagger}(v) e^{i 2 \pi v t} \\
& =\int_{-\infty}^{+\infty} d v \boldsymbol{A}^{\dagger}(-v) e^{-i 2 \pi v t}
\end{aligned}
$$

We then have to solve

$$
\begin{gathered}
(-i 2 \pi v+\gamma / 2) \boldsymbol{A}(v)=-i 2 \pi \delta \boldsymbol{A}(v)+2 \pi \xi \boldsymbol{A}^{\dagger}(-v)+\sqrt{\bar{\gamma} \boldsymbol{b}_{v},} \\
(-i 2 \pi v-\gamma) \boldsymbol{A}(v)=-i 2 \pi \delta \boldsymbol{A}(v)+2 \pi \xi \boldsymbol{A}^{\dagger}(-v) \\
-\sqrt{\gamma} \boldsymbol{B}_{\text {out }}(v) .
\end{gathered}
$$

To simplify the following expressions, we define $\Gamma=$ $\gamma / 4 \pi$. The solution to Eq. (A20) is

$$
\begin{aligned}
\boldsymbol{A}(v)= & \frac{[\Gamma-i(v+\delta)] \sqrt{\Gamma / \pi}}{(\Gamma-i \nu)^{2}+\delta^{2}-|\xi|^{2}} \boldsymbol{b}_{v} \\
& +\frac{\xi \sqrt{\Gamma / \pi}}{(\Gamma-i \nu)^{2}+\delta^{2}-|\xi|^{2}} \boldsymbol{b}_{-v}^{\dagger},
\end{aligned}
$$

and the solution to Eq. (A21) is

$$
\begin{aligned}
\boldsymbol{B}_{\text {out }}(\nu)= & \frac{(\Gamma-i \delta)^{2}+v^{2}+|\xi|^{2}}{(\Gamma-i v)^{2}+\delta^{2}-|\xi|^{2}} \boldsymbol{b}_{v} \\
& +\frac{2 \Gamma \xi}{(\Gamma-i \nu)^{2}+\delta^{2}-|\xi|^{2}} \boldsymbol{b}_{-\nu}^{\dagger} .
\end{aligned}
$$

This is a Bogoliubov relation of the form $\boldsymbol{B}_{\text {out }}(v)=$ $e^{i \phi_{c}} \cosh (\eta) \boldsymbol{b}_{v}+e^{i \phi_{s}} \sinh (\eta) \boldsymbol{b}_{-v}^{\dagger}$, with

$$
\begin{aligned}
\phi_{c}(v, \Gamma,|\xi|, \delta)= & \arctan \left[\frac{2 v \Gamma}{\Gamma^{2}-v^{2}+\delta^{2}-|\xi|^{2}}\right] \\
& -\arctan \left[\frac{2 \delta \Gamma}{\Gamma^{2}-\delta^{2}+v^{2}+|\xi|^{2}}\right], \quad(\mathrm{A} 24) \\
\phi_{S}(v, \Gamma,|\xi|, \delta)= & \arctan \left[\frac{2 v \Gamma}{\Gamma^{2}-v^{2}+\delta^{2}-|\xi|^{2}}\right]+\arg [\xi],
\end{aligned}
$$

$$
\begin{aligned}
& \eta(v, \Gamma,|\xi|, \delta) \\
& \quad=\frac{1}{2} \ln \left[\frac{\sqrt{\left(\Gamma^{2}+v^{2}+|\xi|^{2}-\delta^{2}\right)^{2}+4 \delta^{2} \Gamma^{2}}+2 \Gamma|\xi|}{\sqrt{\left(\Gamma^{2}+v^{2}+|\xi|^{2}-\delta^{2}\right)^{2}+4 \delta^{2} \Gamma^{2}}-2 \Gamma|\xi|}\right] .
\end{aligned}
$$

On the "ridge," for $\delta=0$, this reduces to

$$
\eta(v, \Gamma,|\xi|)=\frac{1}{2} \ln \left[\frac{(\Gamma+|\xi|)^{2}+v^{2}}{(\Gamma-|\xi|)^{2}+v^{2}}\right] .
$$

\section{APPENDIX B: DETECTION MODEL}

At the output of the setup, we measure time-averaged statistics of the band-filtered single mode

$$
\boldsymbol{B}_{h}(t)=\int_{-\infty}^{+\infty} d v h(v) \boldsymbol{B}_{\text {out }}(v) e^{-i 2 \pi v t},
$$

where $h(v)$ is the integral-normalized $\left(\int d v|h(v)|^{2}=1\right)$ filter response function of the detection filter. As $h(v)$ is integral normalized, the quantity $\boldsymbol{N}_{h}(t) \equiv \boldsymbol{B}_{h}^{\dagger}(t) \boldsymbol{B}_{h}(t)$ is the number of photons counted per unit frequency and unit time. The statistical moments of the distribution, which are time independent in the stationary regime, are

$$
\begin{aligned}
\left\langle\boldsymbol{N}_{h}^{k}(t)\right\rangle \equiv & \left\langle n^{k}\right\rangle=\int_{-\infty}^{+\infty} d v_{1} d v_{2} \cdots d v_{2 k-1} d v_{2 k}\left\langle\boldsymbol{B}_{\text {out }}^{\dagger}\left(v_{1}\right) \boldsymbol{B}_{\text {out }}\left(v_{2}\right) \cdots \boldsymbol{B}_{\text {out }}^{\dagger}\left(v_{2 k-1}\right) \boldsymbol{B}_{\text {out }}\left(v_{2 k}\right)\right\rangle \\
& \times h^{*}\left(v_{1}\right) h\left(v_{2}\right) \cdots h^{*}\left(v_{2 k-1}\right) h\left(v_{2 k}\right) \exp \left[i\left(v_{1}-v_{2}+\cdots+v_{2 k-1}-v_{2 k}\right) t\right] .
\end{aligned}
$$


The quantum average is taken on the input mode (in our case, the vacuum) after having substituted all $\boldsymbol{B}_{\text {out }}$ by the appropriate expressions of the free modes $\boldsymbol{b}_{v}$ using Eq. (A23). Applying the usual commutation relations and the effect of the ladder operators on the vacuum, we find that the moments are indeed time independent and verify

$$
\begin{aligned}
\langle n\rangle & =I_{1}, \quad\left\langle\delta n^{2}\right\rangle=I_{1} I_{2}+I_{3}, \\
\left\langle\delta n^{3}\right\rangle & =\left(I_{1}+I_{2}\right)\left(I_{1} I_{2}+3 I_{3}\right),
\end{aligned}
$$

where

$$
I_{1}=\int_{-\infty}^{+\infty} d v|h(v)|^{2} n(v)
$$

with $n(v)=\sinh ^{2}[\eta(v)]$, and

$$
\begin{aligned}
& I_{2}=\int_{-\infty}^{+\infty} d v|h(v)|^{2}[n(v)+1], \\
& I_{3}=\left|\int_{-\infty}^{+\infty} d \nu h(v) h^{*}(-v) \sqrt{n(-v)[n(v)+1]}\right|^{2},
\end{aligned}
$$

where $\eta$ is defined in Eq. (A26).

We can make a few comments about these forms: (1) As $h(v)$ is integral normalized, $I_{2}=I_{1}+1$; and (2) using Cauchy-Schwarz inequality, $I_{3} \leqslant I_{1} I_{2}$, so that $\left\langle\delta n^{2}\right\rangle \leqslant$ $2\langle n\rangle(\langle n\rangle+1)$.

Because of the exponential form of cosh and sinh, it only takes $\eta$ to be of the order of a few units for the CauchySchwarz inequality to become a quasiequality, so that we find, as expected for a squeezed vacuum,

$$
\left\langle\delta n^{2}\right\rangle=2\langle n\rangle(\langle n\rangle+1), \quad\left\langle\delta n^{3}\right\rangle=2\langle n\rangle(2\langle n\rangle+1)(2\langle n\rangle+2) .
$$

In contrast, the statistics presented in Ref. [7] corresponds to frequency-resolved photon detection. The measurement adds up the photocounts of independently resolved orthogonal modes of width $\Delta \rightarrow 0$,

$$
\boldsymbol{B}_{\Delta, n}(t)=\int_{-\infty}^{+\infty} d v \boldsymbol{B}_{\text {out }}(v) \mathcal{D}_{\Delta, n}(v) e^{-i 2 \pi v t},
$$

where we have defined

$$
\mathcal{D}_{\Delta, n}(v) \equiv \mathcal{D}_{\Delta}(v-n \Delta) .
$$

Here, $\mathcal{D}_{\Delta}$ is a function of width $\Delta$, centered around $v=0$, verifying

$$
\int_{-\infty}^{+\infty} d \nu \mathcal{D}_{\Delta}(\nu-n \Delta) \mathcal{D}_{\Delta}(\nu-m \Delta)=\delta_{n, m} .
$$

Examples of mode generator functions are (1) the sinc functions $\mathcal{D}_{\Delta}(v)=\sqrt{\frac{1}{\Delta} \operatorname{sinc}\left(\frac{\pi v}{\Delta}\right)}$ and (2) the window functions $\mathcal{D}_{\Delta}(v)=\frac{1}{\sqrt{\Delta}} \Pi_{\left[-\frac{\Delta}{2} ; \frac{\Delta}{2}\right]}(v)$.

In this theoretical framework, we find

$$
\langle n\rangle_{\Delta}=\sum_{n} J_{1 ; n, n}, \quad\left\langle\delta n^{2}\right\rangle_{\Delta}=\sum_{n, m} J_{1 ; n, m} J_{2 ; n, m}+J_{3 ; n,-m},
$$

where

$$
\begin{aligned}
J_{1 ; n, m} & =\int_{-\infty}^{+\infty} d v \mathcal{D}_{n, \Delta}(v) \mathcal{D}_{m, \Delta}(v) n(v), \\
J_{2 ; n, m} & =\int_{-\infty}^{+\infty} d v \mathcal{D}_{n, \Delta}(v) \mathcal{D}_{m, \Delta}(v)[n(v)+1], \\
J_{3 ; n,-m} & =\left|\int_{-\infty}^{+\infty} d v \mathcal{D}_{n, \Delta}(v) \mathcal{D}_{-m, \Delta}(v) \sqrt{n(-v)[n(v)+1]}\right|^{2} .
\end{aligned}
$$

Using the orthonormality of the $\mathcal{D}_{n, \Delta}(v)$ functions, we have

$$
\int_{-\infty}^{+\infty} d v \mathcal{D}_{\Delta}\left(v-v_{n}\right) \mathcal{D}_{\Delta}\left(v-v_{m}\right) f(v) \simeq \delta_{n, m} f\left(v_{n}\right),
$$

and in the same limit of large $\eta(v)$ as in Eq. (B7), we obtain

$$
\langle n\rangle_{\Delta}=\sum_{n} N_{n}, \quad\left\langle\delta n^{2}\right\rangle_{\Delta}=\sum_{n} 2 N_{n}\left(N_{n}+1\right),
$$

with $N_{n}$ the number of photons in mode $n$.

We can compare these results with those of Eq. (B7), and it becomes apparent that we have a sum of independent modes, centered around their respective frequencies. As a result, we do not find the expected $\left\langle\delta n^{2}\right\rangle=2\langle n\rangle(\langle n\rangle+1)$, as there is an infinite number of modes.

Although always infinite because of the assumed infinite bandwidth, the number of modes increases linearly with the integration time $1 / \Delta$ required to resolve the modes. It is thus a good idea to define rates of photocount moments per unit time, $\Delta\left\langle\delta n^{k}\right\rangle_{\Delta}$, as we can expect them to remain finite. At the same time, we need to take into account the finite bandwidth of the detector, which translates in time domain to an integration time $\tau$ for the detection. The number of photons counted by the detector in any interval of time $\tau$ is thus $\tau \Delta\left\langle\delta n^{k}\right\rangle_{\Delta}$. Taking the limit of $\Delta \rightarrow 0$, we find

$$
\lim _{\Delta \rightarrow 0} \tau \Delta\langle n\rangle_{\Delta}=\tau \int_{-\infty}^{+\infty} d \nu n(\nu)=\gamma \tau F_{n}(\gamma \tau,|\xi| \tau, \delta \tau)
$$

and

$$
\begin{aligned}
\lim _{\Delta \rightarrow 0} \tau \Delta\left\langle\delta n^{2}\right\rangle_{\Delta} & =\tau \int_{-\infty}^{+\infty} d \nu 2 n(v)[n(v)+1] \\
& =\gamma \tau F_{\delta n^{2}}(\gamma \tau,|\xi| \tau, \delta \tau),
\end{aligned}
$$

where $F_{n}$ and $F_{\delta n^{2}}$ are unitless functions of the unitless parameters $\gamma \tau,|\xi| \tau, \delta \tau$. In that sense, they are universal and useful for the characterization of the noise of paramps.

The Fano factor is thus simply

$$
\mathcal{F}=\frac{F_{\delta n^{2}}(\gamma \tau,|\xi| \tau, \delta \tau)}{F_{n}(\gamma \tau,|\xi| \tau, \delta \tau)},
$$

and it depends on the values of the unitless parameters $\Gamma \tau,|\xi| \tau, \delta \tau$.

The Fano factor found in Ref. [7] depends on the value of $\gamma \tau$. It also corresponds to a situation where $\delta=0$, and the free parameter $|\xi| \tau$ (proportional to the pump power) is used as a knob to change the average photon number in the cavity. 


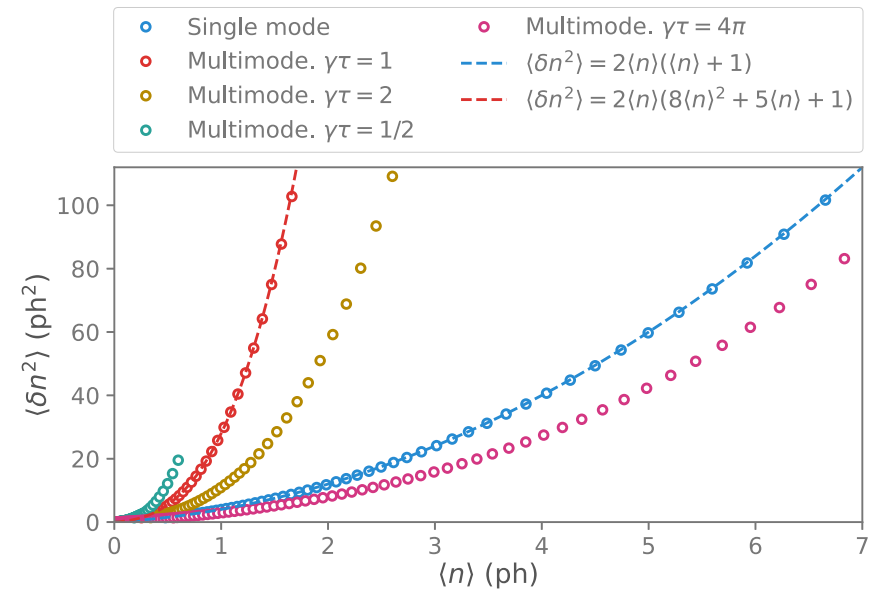

FIG. 4. Comparison between variances for single mode and multimode counting. In our experiment, we count a single mode of the electromagnetic field and the result does not depend on the time of integration. In contrast, in the case of multimode counting, as in Ref. [7], the choice of integration time makes all the difference, as it determines the exact number of modes being counted.

In this specific context we find

$$
\left\langle\delta n^{2}\right\rangle=2\langle n\rangle\left[\left(8 / \gamma^{2} \tau^{2}\right)\langle n\rangle^{2}+(5 / \gamma \tau)\langle n\rangle+1\right],
$$

which is precisely the result obtained in Ref. [7]. Figure 4 illustrates the difference between single-mode counting, always independent of the value of $\gamma \tau$, and multimode counting for various values of $\gamma \tau$.

\section{APPENDIX C: DISCUSSION OF THE MODEL}

Our theoretical analysis clearly explains the importance of the detection scheme and thus why the predictions of Ref. [7] differ from that of the expected squeezed vacuum statistics. It also predicts that with our present setup we should observe the photon statistics of squeezed vacuum. We indeed observe the "right" statistics on the "ridge" of $C_{2}$, that is on the optimal functioning points of the paramp.

However, the theory fails to explain why we observe clouds of points for $\left\langle\delta n^{2}\right\rangle$ and $\left\langle\delta n^{3}\right\rangle$ vs $\langle n\rangle$ in Figs. 3 and 6. It does predict that we can observe a photocount variance lower than that of the squeezed vacuum, but never higher (see Appendix B). One can easily understand that if the measurement bandwidth is finite and not centered on the resonance (i.e., off the ridge) there might be photon pairs which are detected as single photons (the other photon of the pair being outside the detection bandwidth). This leads to a mixture between the squeezed vacuum and thermal state and thus leads to a decrease of $\left\langle\delta n^{2}\right\rangle$. In contrast, we observe that experimental points off the ridge lie both below and above the variance of squeezed vacuum.

In an attempt to correct the theory, we considered the effect of wideband detection [28]. Indeed, the quantity that is detected in our experiments is voltage, the equivalent of the electric field in free space. As shown in Ref. [28], the voltage is not the same thing as the "photonic field" carrying information about photocounts on a detector. So what is really measured is not directly the bosonic mode of Eq. (B1), but

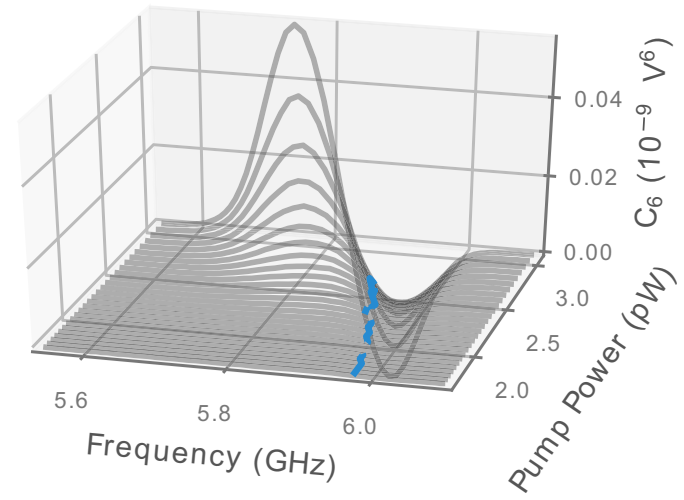

FIG. 5. Measured sixth cumulant of voltage fluctuations generated by the paramp as a function of its resonance frequency and pump power. The dashed blue line corresponds to frequency and pump power equivalent to the blue line of Fig. 2(a).

rather the linked quantity,

$$
\boldsymbol{V}_{h}(t)=\sqrt{\nu_{0}} \int_{-\infty}^{+\infty} d v \sqrt{1+\frac{v}{v_{0}}} h(v) \boldsymbol{B}_{\text {out }}(v) e^{-i 2 \pi v t},
$$

where $v_{0}$ is the central frequency $(6 \mathrm{GHz})$ of the detection band.

We do find a small cloud of points, but they all lie beneath the theoretical maximum variance. In addition, we find a nonzero sixth cumulant $C_{6}$ outside of the ridge, as featured in Fig. 5. This is an interesting feature, as the narrow-band theory predicts $C_{6}=0$ everywhere, just as it is zero on the "ridge" of our experiments. However, the amplitude of the corrected $C_{6}$ is too small by one order of magnitude compared to the experiments. Hence, the wideband correction is insufficient to explain experimental data.

Another potential shortcoming of the theoretical model is the fact that the nonlinear coupling of the Josephson junction is cut at the second order in our Hamiltonian. This is also the

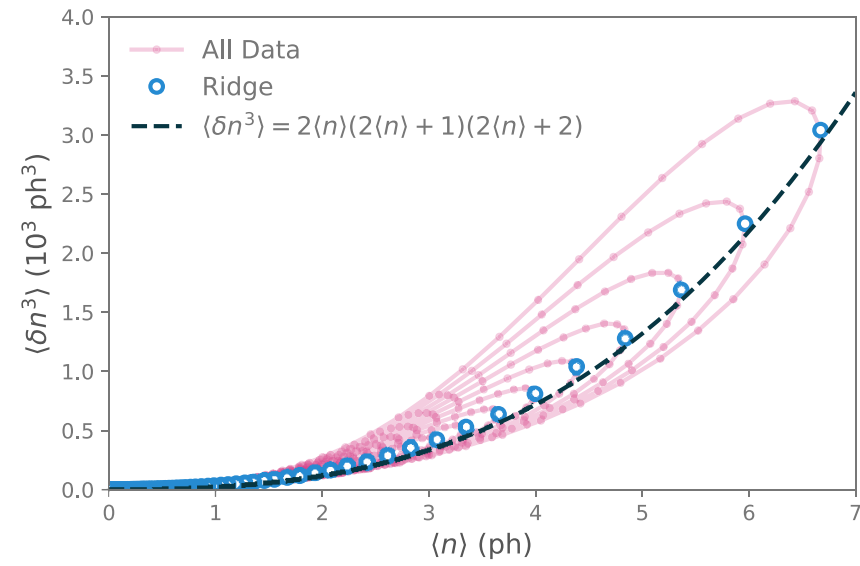

FIG. 6. Skewness of the photocounts $\left\langle\delta n^{3}\right\rangle$ as a function of the average photon number $\langle n\rangle$. Dots are experimental data and each line represents a given pump power. Open circles correspond to the maximum of the paramp gain, i.e., the blue line in Fig. 2. The dashed line corresponds to the theoretical prediction for squeezed vacuum. The results stem from the values of the sixth cumulant of Fig. 5 . 
case for the reference motivating this text [7], so we did not attempt to expand the Hamiltonian to higher orders. However, the Josephson parametric amplifier can be highly nonlinear, and this simplification is likely to fail at higher powers, starting at the single digit photon number. This has been studied in detail in Ref. [26] for the case $\phi=0$. In Figs. 3 and 6, we linked all the points corresponding to the same pump power by a single line. We clearly see that excursions away from the theoretical values increase dramatically with pump power. We also see that the ridge is defined as the maximum of $\langle n\rangle$ versus flux bias for any given pump power, i.e., $\left(\frac{\partial\langle n\rangle}{\partial \Phi}\right)_{P}=0$.
It is straightforward to show that it also corresponds to the minimum pump power for a given $\langle n\rangle$, i.e., $\left(\frac{\partial P}{\partial \Phi}\right)_{\langle n\rangle}=0$. As a consequence, we show that we recover the squeezed vacuum photocount distribution only for a pump power close to the optimum gain (even though our bichromatic pumping scheme is the one that leads to the least nonlinearities [26]). In addition, half the points lie above, and half the points lie below the theoretical curve. Thus we expect that a successful theory would take into account the sign of the flux bias (i.e., it should feature odd terms in the flux bias, which our theory fails to do).
[1] M. A. Castellanos-Beltran, K. D. Irwin, L. R. Vale, G. C. Hilton, and K. W. Lehnert, Bandwidth and dynamic range of a widely tunable Josephson parametric amplifier, IEEE Trans. Appl. Supercond. 19, 944 (2009).

[2] N. Bergeal, F. Schackert, M. Metcalfe, R. Vijay, V. E. Manucharyan, L. Frunzio, D. E. Prober, R. J. Schoelkopf, S. M. Girvin, and M. H. Devoret, Phase-preserving amplification near the quantum limit with a Josephson ring modulator, Nature (London) 465, 64 (2010).

[3] X. Zhou, V. Schmitt, P. Bertet, D. Vion, W. Wustmann, V. Shumeiko, and D. Esteve, High-gain weakly nonlinear fluxmodulated Josephson parametric amplifier using a SQUID array, Phys. Rev. B 89, 214517 (2014).

[4] C. Eichler, Experimental characterization of quantum microwave radiation and its entanglement with a superconducting qubit, Ph.D. thesis, Eidgenössische Technische Hochschule Zürich, 2013, https://doi.org/10.3929/ethz-a-009771047.

[5] S. Jebari, F. Blanchet, A. Grimm, D. Hazra, R. Albert, P. Joyez, D. Vion, D. Estve, F. Portier, and M. Hofheinz, Near-quantumlimited amplification from inelastic Cooper-pair tunnelling, Nat. Electron. 1, 223 (2018).

[6] R. Vyas and S. Singh, Photon-counting statistics of the degenerate optical parametric oscillator, Phys. Rev. A 40, 5147 (1989).

[7] C. Padurariu, F. Hassler, and Y. V. Nazarov, Statistics of radiation at Josephson parametric resonance, Phys. Rev. B 86, 054514 (2012).

[8] S. M. Barnett and P. M. Radmore, Methods in Theoretical Quantum Optics (Oxford University Press, Oxford, U.K., 2005).

[9] J. Huang and P. Kumar, Photon-counting statistics of multimode squeezed light, Phys. Rev. A 40, 1670 (1989).

[10] C. Zhu and C. M. Caves, Photocount distributions for continuous-wave squeezed light, Phys. Rev. A 42, 6794 (1990).

[11] J. W. Goodman, Statistical Optics (Wiley, Hoboken, NJ, 2000).

[12] S. Virally, J. O. Simoneau, C. Lupien, and B. Reulet, Discrete photon statistics from continuous microwave measurements, Phys. Rev. A 93, 043813 (2016).

[13] C. W. Gardiner and M. J. Collett, Input and output in damped quantum systems: Quantum stochastic differential equations and the master equation, Phys. Rev. A 31, 3761 (1985).

[14] M. J. Collett, R. Loudon, and C. W. Gardiner, Quantum theory of optical homodyne and heterodyne detection, J. Mod. Opt. 34, 881 (1987).

[15] C. W. Gardiner and P. Zoller, Quantum Noise, 3rd ed. (Springer, Berlin, 2004).
[16] C. Macklin, K. O’Brien, D. Hover, M. E. Schwartz, V. Bolkhovsky, X. Zhang, W. D. Oliver, and I. Siddiqi, A near-quantum-limited Josephson traveling-wave parametric amplifier, Science 350, 307 (2015).

[17] U. C. Mendes, S. Jezouin, P. Joyez, B. Reulet, A. Blais, F. Portier, C. Mora, and C. Altimiras, Parametric Amplification and Squeezing with an AC- and DC-Voltage Biased Superconducting Junction, Phys. Rev. Appl. 11, 034035 (2019).

[18] M. Hatridge, R. Vijay, D. H. Slichter, J. Clarke, and I. Siddiqi, Dispersive magnetometry with a quantum limited SQUID parametric amplifier, Phys. Rev. B 83, 134501 (2011).

[19] A. Kamal, A. Marblestone, and M. Devoret, Signal-to-pump back action and self-oscillation in double-pump Josephson parametric amplifier, Phys. Rev. B 79, 184301 (2009).

[20] D. F. Santavicca, B. Reulet, B. S. Karasik, S. V. Pereverzev, D. Olaya, M. E. Gershenson, L. Frunzio, and D. E. Prober, Energy resolution of terahertz single-photon-sensitive bolometric detectors, Appl. Phys. Lett. 96, 083505 (2010).

[21] J. Y. Mutus, T. C. White, E. Jeffrey, D. Sank, R. Barends, J. Bochmann, Y. Chen, Z. Chen, B. Chiaro, A. Dunsworth, J. Kelly, A. Megrant, C. Neill, P. J. J. O'Malley, P. Roushan, A. Vainsencher, J. Wenner, I. Siddiqi, R. Vijay, A. N. Cleland et al., Design and characterization of a lumped element single-ended superconducting microwave parametric amplifier with on-chip flux bias line, Appl. Phys. Lett. 103, 122602 (2013).

[22] J. O. Simoneau, S. Virally, C. Lupien, and B. Reulet, Photonpair shot noise in electron shot noise, Phys. Rev. B 95, 060301(R) (2017).

[23] B. Mollow and R. J. Glauber, Quantum theory of parametric amplification. I, Phys. Rev. 160, 1076 (1967).

[24] B. Mollow and R. J. Glauber, Quantum theory of parametric amplification. II, Phys. Rev. 160, 1097 (1967).

[25] M. J. Collett and R. Loudon, Output properties of parametric amplifiers in cavities, J. Opt. Soc. Am. B 4, 1525 (1987).

[26] S. Boutin, D. M. Toyli, A. V. Venkatramani, A. W. Eddins, I. Siddiqi, and A. Blais, Effect of Higher-Order Nonlinearities on Amplification and Squeezing in Josephson Parametric Amplifiers, Phys. Rev. Appl. 8, 054030 (2017).

[27] E. Knill, R. Laflamme, and G. J. Milburn, A scheme for efficient quantum computation with linear optics, Nature (London) 409, 46 (2001).

[28] S. Virally and B. Reulet, Unidimensional time-domain quantum optics, Phys. Rev. A 100, 023833 (2019). 